\title{
Nonalcoholic Fatty Liver Disease (NAFLD) and Risk of Cardiac Arrhythmias: A New Aspect of the Liver-heart Axis
}

\author{
Alessandro Mantovani* \\ Section of Endocrinology, Diabetes and Metabolism, Department of Medicine, University and \\ Azienda Ospedaliera Universitaria Integrata of Verona, Verona, Italy
}

\begin{abstract}
Nonalcoholic fatty liver disease (NAFLD) is a pathologic condition frequently observed in clinical practice. To date, the prevalence of NAFLD is approximately $25-30 \%$ among adults of the general population in Western countries but increases to approximately $70-75 \%$ among patients with type 2 diabetes mellitus. In the last decade, accumulating evidence has clearly demonstrated that patients with NAFLD have not only an increased liver-related morbidity and mortality but also an increased risk of fatal and non-fatal cardiovascular events. In particular, several studies have documented the existence of an independent association among NAFLD and cardiac changes in structure and function in both non-diabetic and diabetic patients. In addition, mounting evidence also suggests that there is a strong relationship between NAFLD and cardiac arrhythmias, such as atrial fibrillation, QTc prolongation and ventricular arrhythmias. This is of clinical interest, as it could explain, at least in part, the increased risk of death for cardiovascular disease in patients with NAFLD. Therefore, seeing that cardiovascular disease complications are the leading cause of disability and death in NAFLD patients, the recent European clinical practice guidelines advised to check the cardiovascular system in all patients with NAFLD. This clinical mini review will briefly describe the increasing body of evidence regarding the association between NAFLD and cardiac arrhythmias, and discuss the potential biological mechanisms underlying this association.

Citation of this article: Mantovani A. Nonalcoholic fatty liver disease (NAFLD) and risk of cardiac arrhythmias: a new aspect of the liver-heart axis. J Clin Transl Hepatol 2017;5(2):134-141. doi: 10.14218/JCTH.2017.00005.
\end{abstract}

\footnotetext{
Keywords: Nonalcoholic fatty liver disease; Cardiovascular disease; Cardiac arrhythmias; Cardiac complications.

Abbreviations: AF, atrial fibrillation; ALT, alanine aminotransferase; APCs, atrial premature complexes; AST, aspartate aminotransferase; AV, atrioventricular; BMI, body mass index; COPD, chronic obstructive pulmonary disease; CHD, coronary heart disease; CKD, chronic kidney disease; ECG, electrocardiogram; ESRD, end-stage renal disease; GGT, gamma-glutamyltransferase; HF, heart failure; IHD, ischemic heart disease; LA, left atrium; LV, left ventricular; LVEF, left ventricular ejection fraction; MetS, metabolic syndrome; PVCs, premature ventricular complexes; RBBB, right bundle brunch block; T1DM, type 1 diabetes mellitus; T2DM, type 2 diabetes mellitus; US, ultrasonography; VHD, valvular heart disease; VT, ventricular tachycardia.

Received: 16 January 2017; Revised: 29 March 2017; Accepted: 30 March 2017 *Correspondence to: Alessandro Mantovani, Section of Endocrinology, Diabetes and Metabolism, Department of Medicine, University and Azienda Ospedaliera Universitaria Integrata, Piazzale Stefani, 1, 37126 Verona, Italy. Tel: +39-458123110, Fax: +39-45-8122841, E-mail: alessandro.mantovani24@gmail.com
}

\section{Introduction}

Nonalcoholic fatty liver disease (NAFLD) is currently a very common disease in Western countries. ${ }^{1}$ The prevalence of NAFLD is approximately $25-30 \%$ among the adult general population but increases to $70-75 \%$ among patients with type 2 diabetes (T2DM) and to $95-99 \%$ among patients with obesity. ${ }^{1-3}$ NAFLD is currently the second most common indication for liver transplantation in the United States, and there is a worrying tendency that it will become the first in the near future. ${ }^{1-3}$ of note, patients with T2DM have a greater risk of developing the more severe forms of NAFLD, including nonalcoholic steatohepatitis (NASH), cirrhosis and hepatocellular carcinoma. ${ }^{1-4}$

In the last decade, it has become increasing clear that NAFLD is not only associated with an increased liver-related morbidity and mortality but that coronary heart disease is the primary cause of death in patients with NAFLD. ${ }^{4-6}$ Convincing data have also indicated the existence of a strong association between NAFLD and alterations in cardiac function and structure in patients with and without type 2 diabetes, independent of overweight/obese status and presence of hypertension or other features of metabolic syndrome. ${ }^{4-6}$ Moreover, several observational studies have also demonstrated that patients with NAFLD have an increased risk of cardiac arrhythmias, including atrial fibrillation (AF), heart rate-corrected QT (QTC) interval prolongation and ventricular arrhythmias. ${ }^{6}$

This research field is of clinical interest as it could explain, at least in part, the increased risk of cardiovascular mortality that is observed in patients with NAFLD. Seeing that cardiovascular disease complications recurrently drive the outcomes of patients with NAFLD, the recent European clinical practice guidelines for the management of NAFLD has recommended screening of the cardiovascular system for all patients with NAFLD, at least by detailed risk factor evaluation. $^{7}$

This clinical mini review will describe the large body of evidence supporting the existence of a strong association between NAFLD and arrhythmic complications and will discuss the putative biological mechanisms through which NAFLD might contribute to the physiopathology of cardiac arrhythmias.

\section{Definition, diagnosis and prevalence of NAFLD}

The spectrum of NAFLD includes a variety of progressive liver diseases, ranging from simple steatosis (histologically characterized by infiltration in $>5 \%$ of hepatocytes) to nonalcoholic steatohepatitis or NASH (histologically indicated by fatty 
infiltration, balloon degeneration and inflammation with and without fibrosis) as well as cirrhosis, that may, in some cases, also progress to hepatocellular carcinoma., 8 Diagnosis of NAFLD is always a diagnosis of exclusion and is formulated according to the following criteria: (1) identification of hepatic steatosis by imaging or histology, and (2) exclusion of secondary known causes of chronic liver disease, including alcohol (a threshold of $30 \mathrm{~g} /$ day for men and $20 \mathrm{~g} /$ day for women is conventionally adopted), virus, drugs, autoimmunity and hemochromatosis. ${ }^{7,8}$

The precise prevalence of NAFLD is not known, due to at least two reasons. First, the prevalence of NAFLD can vary through the people that are studied, according to age, sex, ethnicity and various comorbidities. Second, the prevalence of NAFLD can vary widely according to the different approaches used for its detection; these include serum liver enzyme assay, imaging analysis (i.e. ultrasonography or magnetic resonance) and histological analysis. That being said, as recently reported by a meta-analysis of Younossi et al., ${ }^{1}$ it is generally estimated that $30-35 \%$ of adult North Americans have NAFLD (as detected by ultrasonography), whereas among Europeans and the Middle Easterners the prevalence of NAFLD ranges from $20 \%$ to $30 \%{ }^{2,9}$ Prevalence of NALFD among the Japanese and Chinese similarly ranges from $20-30 \%$ and $15-30 \%$, respectively. ${ }^{10-12}$ For populations living on the Indian subcontinent, the prevalence ranges from $16 \%$ to $32 \% .{ }^{13}$

It is important to highlight that in high-risk populations (i.e. patients with T2DM, obesity, dyslipidemia or hypertension), the prevalence of NAFLD is much higher. ${ }^{3,14}$ For example, the reported prevalence of NAFLD detected by ultrasonography in patients with T2DM has ranged from $45 \%$ to $75 \%$ in large hospital-based studies and from $30 \%$ to $70 \%$ in population-based studies. ${ }^{14}$

\section{Epidemiological evidence linking NAFLD to cardiac arrhythmias}

Given the strong relationship that has been observed between NAFLD and traditional and non-traditional cardiovascular risk factors, many observational studies have investigated the role of NAFLD on the risk of developing cardiac arrhythmias, including AF, QTC prolongation and ventricular arrhythmias. Table 1 summarizes the main observational studies that have reported an association between NAFLD and risk of cardiac arrhythmias.

\section{NAFLD and risk of supraventricular arrhythmias}

AF is the most common supraventricular arrhythmia seen in clinical practice. ${ }^{15}$ As the aging population increases and cardiovascular treatments continue to improve, however, it is thought that the prevalence and incidence of AF will rise, likely substantially. ${ }^{15}$ While the first description of simultaneous presence of peripheral artery disease and $A F$ in a patient with hepatic steatosis and T2DM was reported more than 60 years ago, ${ }^{16}$ only few studies to date have investigated the role of NAFLD as a risk factor of AF.

In 2013, in a longitudinal study involving more than 3,700 white adults free of AF at baseline, the Framingham Heart Study Researchers showed that increased levels of serum transaminases (i.e. alanine aminotransferase [ALT] and aspartate aminotransferase [AST]) were independently associated with increased risk of incident AF over 10 years of follow-up. ${ }^{17}$ Interestingly, these associations remained significant after adjustment for multiple AF risk factors and other potential confounders. ${ }^{17}$ Almost identical findings were reported by Alonso et al. ${ }^{18}$ from another large populationbased study; specifically, moderately elevated gammaglutamyltransferase (GGT) levels (i.e. a marker of NAFLD) were found to be strongly associated with an increased incidence of AF over a mean period of 12 years. This association also persisted after adjustment for several cardio-metabolic factors and other potential confounding variables. ${ }^{18}$

Recently, a prospective study by Käräjämäki et al. ${ }^{19}$ involving approximately 1,000 middle-aged Finnish individuals with a mean follow-up period of 16 years found that NAFLD, as detected by ultrasonography, was associated with a 2-fold increased risk of incident AF. Moreover, this association was independent of age, sex, body mass index, waist circumference, alcohol consumption, smoking, blood pressure, diabetes status, ALT levels, insulin resistance, atrial natriuretic peptide levels, C-reactive protein levels and relevant echocardiographic parameters.

Almost identical findings were observed in patients with T2DM. ${ }^{20,21}$ In a cross-sectional study including 702 hospitalized T2DM patients with no cancer and end-renal stage disease, Targher et al. ${ }^{20}$ showed that patients with NAFLD, as detected by ultrasound, had a higher prevalence of permanent/persistent $\mathrm{AF}$ than their counterparts without liver involvement. In the multivariate regression analysis, this association between NAFLD and risk of prevalent permanent/persistent AF was found to be independent of several cardiovascular risk factors, diabetes-related variables and other potential confounders. ${ }^{20}$ In addition, when patients were stratified simultaneously by the median value of serum GGT levels and the NAFLD status on ultrasonography, those with hepatic steatosis, regardless of their serum GGT level, showed the highest prevalence of permanent/persistent $A F^{20}$ In the authors' subsequent longitudinal study involving 400 outpatients with T2DM who were free of pre-existent AF at baseline and followed up for a mean period of 10 years, the patients with NAFLD showed a higher risk of developing new-onset AF than those without NAFLD. ${ }^{21}$ This association was also found to be independent of age, sex, hypertension, PR interval on electrocardiogram, left ventricular hypertrophy and other relevant variables included in the 10-year Framingham Heart Study-derived AF risk score. ${ }^{21}$

Currently, there is not accurate information regarding the potential underlying biological mechanisms that link NAFLD to an increased risk of AF in patients with NAFLD. Fig. 1 shows the putative biological mechanisms that might be implicated. It is well known that abnormal atrial conduction can play a crucial role in the pathophysiology of AF. However, the current literature includes only a single small study assessing atrial conduction characteristics in patients with NAFLD. ${ }^{22}$ Interestingly, in that case-control study, Ozveren et $a .^{22}$ found that patients with NAFLD who were free from hypertension, diabetes or known cardiac diseases had longer inter-atrial and intra-atrial electromechanical delay intervals (as detected by tissue Doppler echocardiography) and higher $\mathrm{P}$-wave dispersion compared to those without liver involvement, suggesting the existence of an impaired atrial conduction in patients with NAFLD. No studies to date have reported an association between NAFLD and other atrial arrhythmias, such as atrial premature complexes (APCs) and paroxysmal supraventricular tachyarrhythmia. However, it is important to emphasize that, in a recent cross-sectional study that 


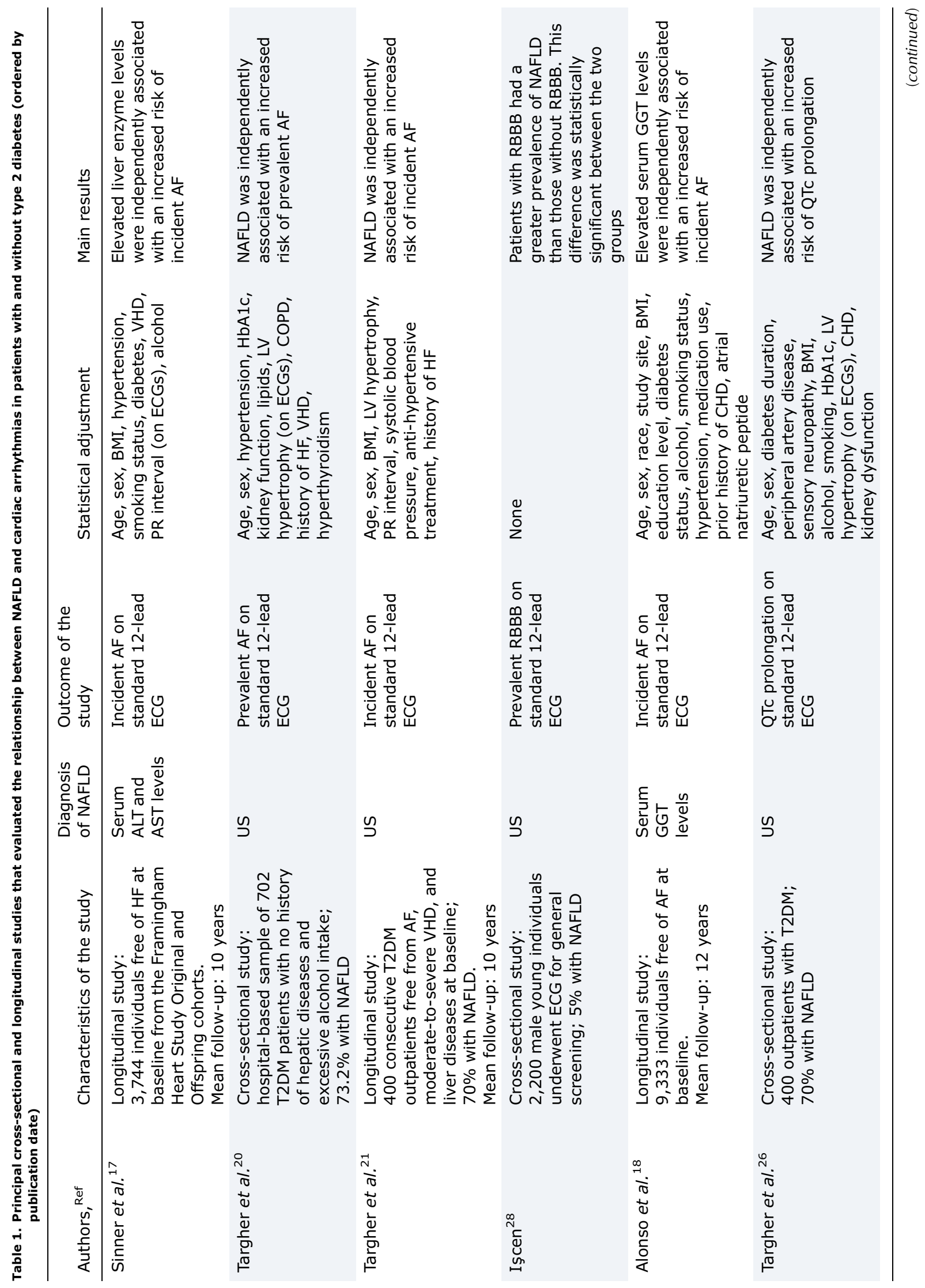




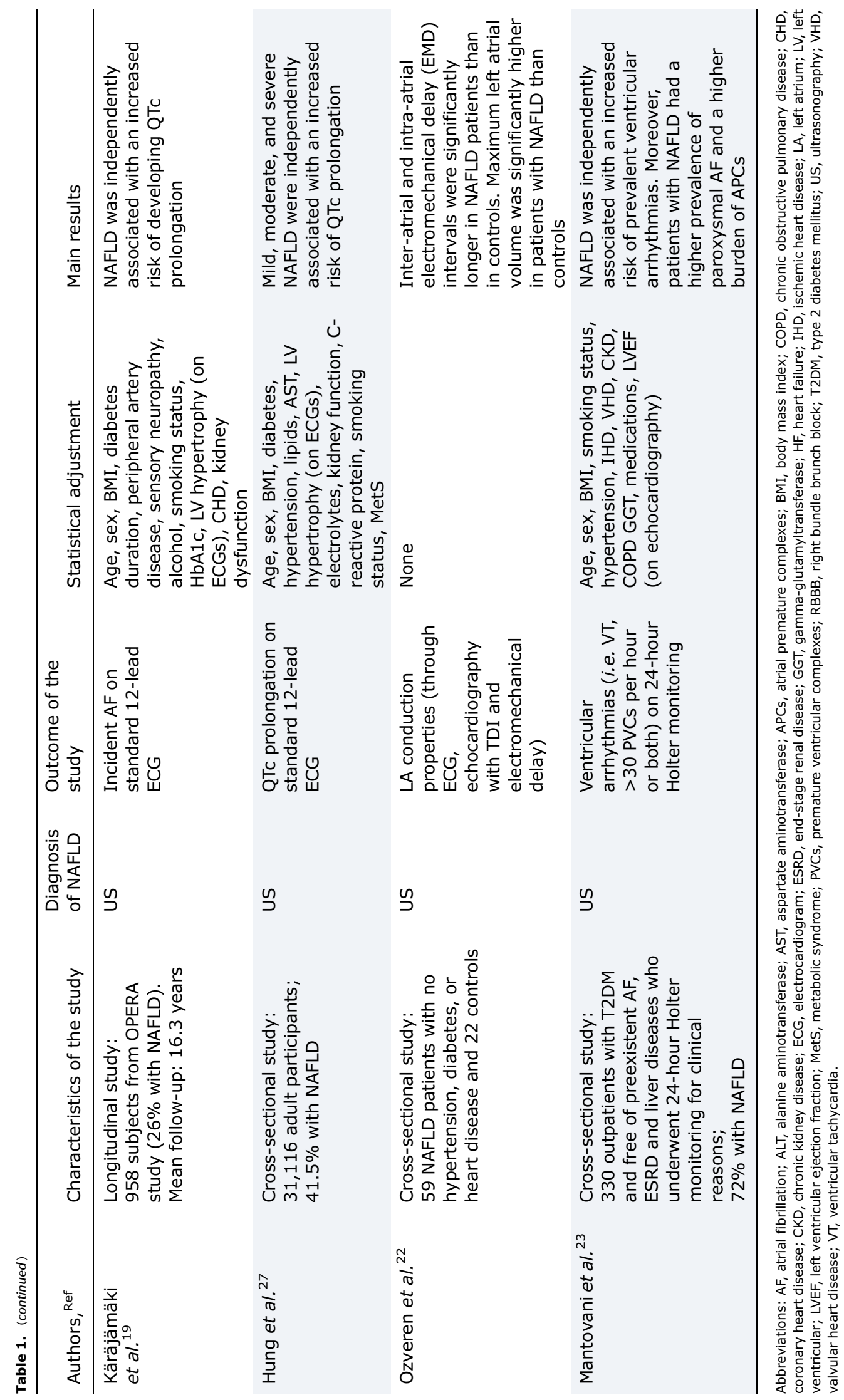


included more than 300 outpatients with T2DM who had undergone 24-hour Holter monitoring, Mantovani et al. ${ }^{23}$ found that patients with NAFLD had a higher prevalence of paroxysmal AF and a higher burden of APCs compared to those without liver involvement.

\section{NAFLD and risk of ventricular arrhythmias}

Emerging research suggests an association between NAFLD and QTc interval prolongation on standard 12-lead electrocardiogram (ECG). It is well known that QTc interval prolongation is a powerful risk factor for ventricular arrhythmias and is also associated with an increased risk of cardiovascular mortality and sudden cardiac death in patients with and without diabetes mellitus. ${ }^{24,25}$ Therefore, evaluation of a possible relationship between NAFLD and QTc prolongation is of great interest in clinical practice.

A cross-sectional study including 400 outpatients with T2DM and without a documented history of AF showed that moderate-to-severe heart valve diseases, hepatic disease, alcohol consumption, and the presence and severity of NAFLD on ultrasound were associated with an approximately 2-fold higher risk of prolonged QTc interval duration, independent of age, sex, hypertension, diabetes-related variables and other well-known cardiovascular risk factors. ${ }^{26}$ In addition, the authors demonstrated a significant and graded relationship between severity of NAFLD and frequency of patients with QTc interval prolongation. ${ }^{26}$ In a recent study, Hung et al. ${ }^{27}$ investigated whether the association between NAFLD and QTC interval prolongation also exists in the general adult population. In that study, the investigators reported that the severity of NAFLD was associated with a higher risk of QTC interval prolongation, even after adjustment for several cardiometabolic risk factors and other important comorbidities. ${ }^{27}$ Notably, such association was found to be present in all subgroups of patients analyzed, including patients with T2DM. ${ }^{27}$

More recently, in a cross-sectional study involving 330 outpatients with T2DM and without pre-existing $A F$, end-stage renal disease or known liver diseases, who underwent 24-hour ambulatory Holter monitoring for clinical reasons, Mantovani et al. ${ }^{23}$ found that NAFLD diagnosed by ultrasound was associated with an approximately 3-fold increased risk of ventricular arrhythmias (defined as presence of non-sustained ventricular tachyarrhythmia, $\geq 30$ premature ventricular complexes per hour, or both). Notably, this association remained significant even after adjusting for age, sex, body mass index, hypertension, smoking status, LV ejection fraction, medications, prior history of ischemic heart disease, diabetic neuropathy, chronic kidney disease and other important comorbidities. ${ }^{23}$

Collectively, even though the arrhythmogenic role of NAFLD requires further confirmation in larger longitudinal studies, this research field can be highly promising. Indeed,

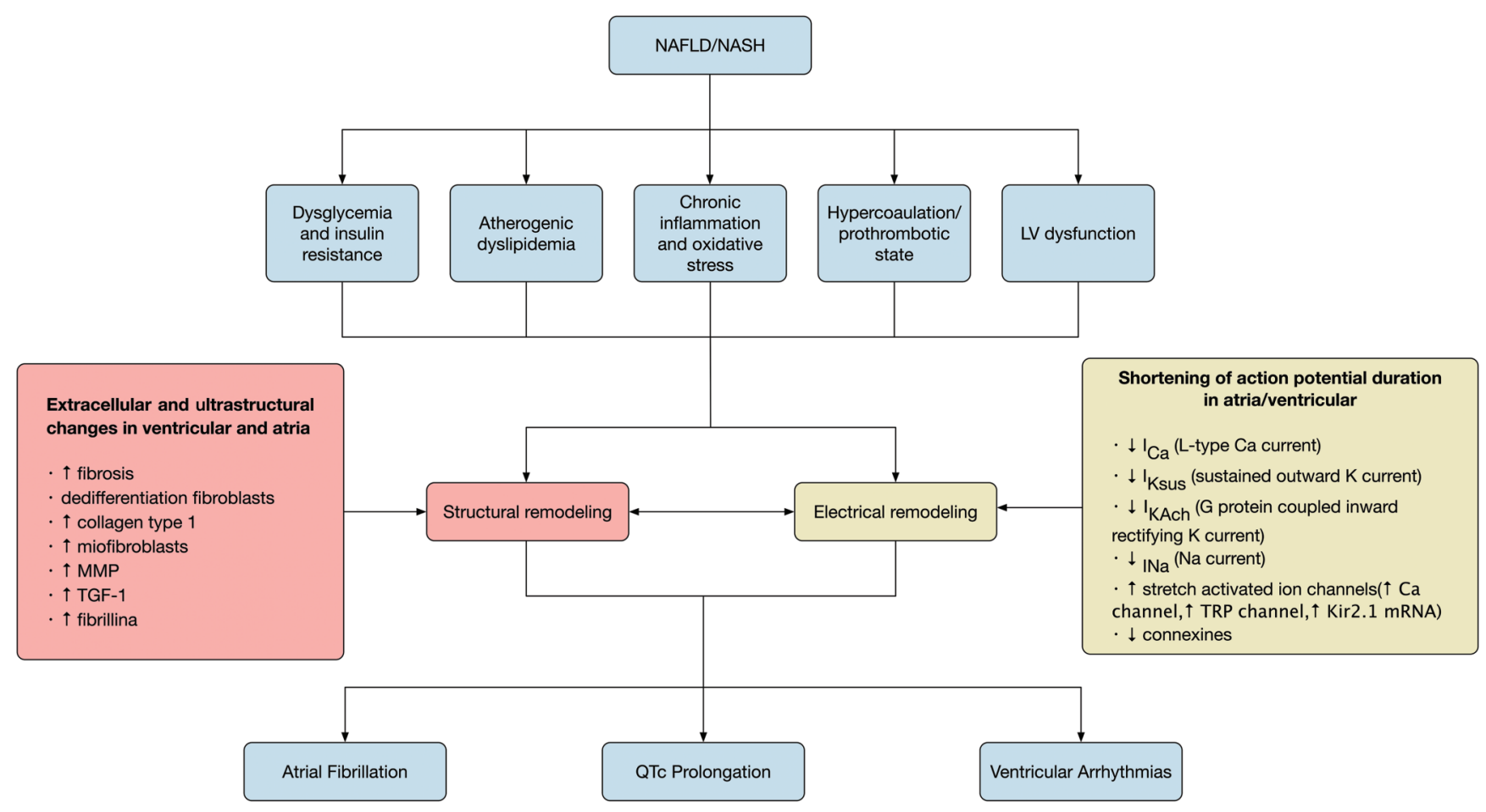

Fig. 1. Putative biological mechanisms linking NAFLD and risk of cardiac arrhythmias (i.e. atrial fibrillation, QTc interval prolongation and ventricular arrhythmias). The pathophysiological mechanisms linking NAFLD to cardiac arrhythmias are complex and not completely understood. In the presence of NAFLD (or NASH), several alterations occur in the liver, resulting in an increased production of atherogenic lipids (e.g., very-low-density lipoproteins, small and dense low-density lipoproteins, and non-esterified fatty acids) and in an increased release of many pro-inflammatory (e.g., c-reactive protein, tumor necrosis factor-alpha, and interleukin-6), pro-fibrinogen (transforming growth factor-beta), pro-oxidant, vasoactive and thrombogenic (e.g., factor VIII, plasminogen activator inhibitor-1, and endotelin-1) molecules. These NAFLD-related alterations might have adverse effects on the risk of cardiac arrhythmias. In particular, it is well known that pro-inflammatory and pro-oxidant mediators are able to alter electrophysiology and structural substrates of myocardium, leading to increased vulnerability to cardiac arrhythmias. For instance, many inflammatory cytokines (i.e. tumor necrosis factor-alpha, interleukin-1, and interleukin-6) can modulate calcium homeostasis and connexins, that are associated with modifications in fiber continuity and possible circuit re-entry, and also promote myolysis, cardiomyocyte apoptosis and myocardial fibrogenesis. 
the pathophysiological mechanisms implicated in the contribution per se of NAFLD to insulin resistance and chronic inflammation might represent potential new pharmacological targets for the prevention of myocardial alterations in patients with NAFLD.

\section{NAFLD and risk of bundle branch and atrioventricular (AV) blocks}

Currently, only a single observational study assessing the relationship between NAFLD and risk of bundle branch blocks has been reported in literature. ${ }^{28}$ In general, bradycardia can occur when impulse conduction across the AV node is compromised, and this is accompanied by worrying possibilities of concomitant symptoms (e.g., fatigue or syncope) and even death, especially when ventricular rates are completely ineffective. Transient AV conduction block is common in young people and is most likely due to high vagal tone. Acquired and persistent failure of $\mathrm{AV}$ conduction is infrequent in healthy adults, but becomes common in the setting of aging, atherosclerosis, myocardial ischemia, diabetes, or autoimmune and infiltrative diseases.

While AV blocks of high-degrees (i.e. second- and thirddegree) predict development of cardiovascular events in affected patients, recent accumulating evidence also suggests that even prolonged PR interval, first-degree AV block and bundle branch blocks are associated with adverse outcomes, independent of well-known cardiovascular risk factors. ${ }^{29-36}$ In a pivotal cross-sectional study involving more than 2,000 male young subjects who underwent a standard 12-lead ECG for general young health screening, Işcen ${ }^{28}$ found that the presence of right bundle branch block was significantly associated with an increased prevalence of NAFLD on ultrasonography. Certainly, more studies are needed to confirm this finding and to better explain whether NAFLD is also associated with an increased risks of first-degree AV block, second-degree AV block, third-degree $\mathrm{AV}$ block and other bundle branch blocks.

Finally, more research is also required to identify the biological mechanisms through which NAFLD (or NASH) might promote fibrosis of myocardium and derangements of the HisPurkinje system, inducing a delay of the impulse conduction across the AV node, His bundle, and bundle branches.

\section{Putative biological mechanisms linking NAFLD and cardiac arrhythmias}

To date, the exact biological mechanisms linking the relationship between NAFLD and cardiac arrhythmias are not completely understood. Certainly, the biological mechanisms relating NAFLD, inflamed visceral adipose tissue and altered gut microbiota with cardiac complications are very complex. As demonstrated by several experimental studies, inflamed adipose tissue and intestinal dysbiosis may influence the development and progression of NAFLD, through the production of pro-inflammatory molecules, non-esterified and short-chain fatty acids and the reduction of the levels of adiponectin. 4,6,37,38

When NAFLD occurs, liver fat/inflammation progresses (NASH) and advanced fibrosis develops. Many alterations take place into the liver, resulting in an increased production of atherogenic lipids (e.g., very-low-density lipoproteins, small and dense low-density lipoproteins, and non-esterified fatty acids) and in an increased release into bloodstream of several pro-inflammatory (e.g., c-reactive protein, tumor necrosis factor-alpha, and interleukin-6), pro-fibrinogen (e.g., transforming growth factor-beta), pro-oxidant and thrombogenic (e.g., factor VIII, plasminogen activator inhibitor-1, and endotelin-1) mediators. 4,6,39-42 These NAFLDrelated molecules might have a negative impact on the risk of cardiac complications, including arrhythmias. ${ }^{6,37,38}$ In particular, the derangements of the myocardium might potentially produce cellular and ultrastructural changes (e.g., fibrosis and dedifferentiation of fibroblasts) in myocardium as well as alterations of the action potential duration (e.g., modifications of ion current and decreased levels of connexins), resulting in an increased risk of cardiac arrhythmias and rhythm disturbances.

Furthermore, accumulating evidence also suggest a role of NAFLD in the alterations in myocardial function and structure, including heart valve calcifications, early left ventricular diastolic dysfunction and left ventricular hypertrophy. ${ }^{43-48}$ These NAFLD-related myocardium alterations might induce deep changes in fiber continuity and possible circuit re-entry, contributing to the electrophysiological disturbance. Finally, preliminary data also suggest that NAFLD is associated with cardiac autonomic dysfunction, which is a powerful risk factor for ventricular arrhythmias. ${ }^{49} \mathrm{Fig}$. 1 illustrates the putative biological mechanisms linking NAFLD to cardiac arrhythmias.

\section{Conclusions}

The view that NAFLD is a benign disease with a good prognosis and little clinical significance has been changing over the last years. Currently, NAFLD diagnosis is very common, especially among patients with obesity and T2DM, and has become a relevant health care problem worldwide, as it is an important cause of liver-related and cardiovascular mortality and morbidity. Indeed, mounting evidence clearly demonstrates that NAFLD is strongly associated with cardiac complications, including arrhythmias (i.e. AF, QTc prolongation and ventricular arrhythmia), independent of the coexistence of well-known cardiovascular risk factors and features of metabolic syndrome. These findings may explain, at least in part, the increased risk of cardiovascular death observed in patients with NAFLD. As also recently suggested by European clinical practice guidelines, a multidisciplinary approach to NAFLD patients is necessary, based on careful assessment of cardio-metabolic risk factors and regular monitoring of liver and cardiovascular complications. However, much research is required to better understand the pathophysiological mechanisms underlying the association between NAFLD and cardiac arrhythmias and to identify the potential therapeutic targets to prevent the myocardium derangements induced by NAFLD. Finally, the clinical uncertainty of whether the role of NAFLD in the development of cardiac arrhythmias is restricted to NASH and advanced fibrosis or is also associated with simple steatosis remains unanswered.

\section{Conflict of interest}

The author has no conflict of interests related to this publication.

\section{Author contributions}

Reviewed the literature and wrote the manuscript (AM). 


\section{References}

[1] Younossi ZM, Koenig AB, Abdelatif D, Fazel Y, Henry L, Wymer M. Global epidemiology of nonalcoholic fatty liver disease-Meta-analytic assessment of prevalence, incidence, and outcomes. Hepatology 2016;64:73-84. doi: 10.1002/hep.28431.

[2] Non-alcoholic Fatty Liver Disease Study Group, Lonardo A, Bellentani S, Argo CK, Ballestri S, Byrne CD, et al. Epidemiological modifiers of non-alcoholic fatty liver disease: Focus on high-risk groups. Dig Liver Dis 2015;47: 997-1006. doi: 10.1016/j.dld.2015.08.004.

[3] Portillo-Sanchez P, Bril F, Maximos M, Lomonaco R, Biernacki D, Orsak B, et al. High prevalence of nonalcoholic fatty liver disease in patients with type 2 diabetes mellitus and normal plasma aminotransferase levels. J Clin Endocrinol Metab 2015;100:2231-2238. doi: 10.1210/jc.2015-1966.

[4] Targher G, Day CP, Bonora E. Risk of cardiovascular disease in patients with nonalcoholic fatty liver disease. N Engl J Med 2010;363:1341-1350. doi: 10.1056/NEJMra0912063.

[5] Targher G, Byrne CD, Lonardo A, Zoppini G, Barbui C. Non-alcoholic fatty liver disease and risk of incident cardiovascular disease: A meta-analysis. J Hepatol 2016;65:589-600. doi: 10.1016/j.jhep.2016.05.013.

[6] Mantovani A, Ballestri S, Lonardo A, Targher G. Cardiovascular disease and myocardial abnormalities in nonalcoholic fatty liver disease. Dig Dis Sci 2016;61:1246-1267. doi: 10.1007/s10620-016-4040-6.

[7] European Association for the Study of the Liver (EASL); European Association for the Study of Diabetes (EASD); European Association for the Study of Obesity (EASO). EASL-EASD-EASO Clinical Practice Guidelines for the management of non-alcoholic fatty liver disease. J Hepatol 2016;64:1388-1402. doi: $10.1016 / j$.jhep.2015.11.004.

[8] Chalasani N, Younossi Z, Lavine JE, Diehl AM, Brunt EM, Cusi K, et al. The diagnosis and management of non-alcoholic fatty liver disease: practice guideline by the American Gastroenterological Association, American Association for the Study of Liver Diseases, and American College of Gastroenterology. Gastroenterology 2012;142:1592-1609. doi: 10.1053/j.gastro. 2012. 04.001.

[9] Loomba R, Sanyal AJ. The global NAFLD epidemic. Nat Rev Gastroenterol Hepatol 2013;10:686-690. doi: 10.1038/nrgastro.2013.171.

[10] Fan JG, Jia JD, Li YM, Wang BY, Lu LG, Shi JP, et al. Guidelines for the diagnosis and management of nonalcoholic fatty liver disease: update 2010: (published in Chinese on Chinese Journal of Hepatology 2010; 18:163166). J Dig Dis 2011;12:38-44. doi: 10.1111/j.1751-2980.2010.00476.x.

[11] Farrell GC, Wong VW, Chitturi S. NAFLD in Asia-as common and important as in the West. Nat Rev Gastroenterol Hepatol 2013;10:307-318. doi: 10. 1038/nrgastro.2013.34.

[12] Das K, Das K, Mukherjee PS, Ghosh A, Ghosh S, Mridha AR, et al. Nonobese population in a developing country has a high prevalence of nonalcoholic fatty liver and significant liver disease. Hepatology 2010;51:1593-1602. doi: 10.1002/hep.23567.

[13] Singh SP, Nayak S, Swain M, Rout N, Mallik RN, Agrawal O, et al. Prevalence of nonalcoholic fatty liver disease in coastal eastern India: a preliminary ultrasonographic survey. Trop Gastroenterol 2004;25:76-79.

[14] Targher G, Byrne CD. Clinical Review: Nonalcoholic fatty liver disease: a novel cardiometabolic risk factor for type 2 diabetes and its complications. J Clin Endocrinol Metab 2013;98:483-495. doi: 10.1210/jc.2012-3093.

[15] Rahman F, Kwan GF, Benjamin EJ. Global epidemiology of atrial fibrillation. Nat Rev Cardiol 2014;11:639-654. doi: 10.1038/nrcardio.2014.118.

[16] Diabetes mellitus; auricular fibrillation; arteriosclerosis obliterans of the legs; gangrene of the 1 st and $2 \mathrm{~d}$ toes of the right foot; fatty degeneration of the liver. Arq Bras Med 1952;42:212-216.

[17] Sinner MF, Wang N, Fox CS, Fontes JD, Rienstra M, Magnani JW, et al. Relation of circulating liver transaminase concentrations to risk of newonset atrial fibrillation. Am J Cardiol 2013;111:219-224. doi: 10.1016/j. amjcard.2012.09.021.

[18] Alonso A, Misialek JR, Amiin MA, Hoogeveen RC, Chen LY, Agarwal SK, et al. Circulating levels of liver enzymes and incidence of atrial fibrillation: the Atherosclerosis Risk in Communities cohort. Heart 2014;100:1511-1516. doi: 10.1136/heartjnl-2014-305756.

[19] Käräjämäki AJ, Pätsi OP, Savolainen M, Kesäniemi YA, Huikuri H, Ukkola O. Non-alcoholic fatty liver disease as a predictor of atrial fibrillation in middleaged population (OPERA study). PLoS One 2015;10:e0142937. doi: 10. 1371 /journal.pone.0142937.

[20] Targher G, Mantovani A, Pichiri I, Rigolon R, Dauriz M, Zoppini G, et al. Nonalcoholic fatty liver disease is associated with an increased prevalence of atrial fibrillation in hospitalized patients with type 2 diabetes. Clin Sci (Lond) 2013;125:301-309. doi: 10.1042/CS20130036.

[21] Targher G, Valbusa F, Bonapace S, Bertolini L, Zenari L, Rodella S, et al. Nonalcoholic fatty liver disease is associated with an increased incidence of atrial fibrillation in patients with type 2 diabetes. PLoS One 2013;8:e57183. doi: 10.1371 journal.pone.0057183.
[22] Ozveren O, Izgi C, Eroglu E, Simsek MA, Turer A, Kucukdurmaz Z, et al. Doppler tissue evaluation of atrial conduction properties in patients with non-alcoholic fatty-liver disease. Ultrason Imaging 2016;38:225-235. doi: $10.1177 / 0161734615595015$.

[23] Mantovani A, Rigamonti A, Bonapace S, Bolzan B, Pernigo M, Morani G, et al. Nonalcoholic fatty liver disease is associated with ventricular arrhythmias in patients with type 2 diabetes referred for clinically indicated 24-hour holter monitoring. Diabetes Care 2016;39:1416-1423. doi: 10. 2337/dc16-0091.

[24] Straus SM, Kors JA, De Bruin ML, van der Hooft CS, Hofman A, Heeringa J, et al. Prolonged QTc interval and risk of sudden cardiac death in a population of older adults. J Am Coll Cardiol 2006;47:362-367. doi: 10.1016/j.jacc. 2005.08.067.

[25] Okin PM, Devereux RB, Lee ET, Galloway JM, Howard BV; Strong Heart Study. Electrocardiographic repolarization complexity and abnormality predict all-cause and cardiovascular mortality in diabetes: the strong heart study. Diabetes 2004;53:434-440. doi: 10.2337/diabetes.53.2.434.

[26] Targher G, Valbusa F, Bonapace S, Bertolini L, Zenari L, Pichiri I, et al. Association of nonalcoholic fatty liver disease with QTC interval in patients with type 2 diabetes. Nutr Metab Cardiovasc Dis 2014;24:663-669. doi: 10. 1016/j.numecd.2014.01.005.

[27] Hung CS, Tseng PH, Tu CH, Chen CC, Liao WC, Lee YC, et al. Nonalcoholic fatty liver disease is associated with QT prolongation in the general population. J Am Heart Assoc 2015;4:e001820. doi: 10.1161/JAHA.115. 001820.

[28] Isscen S. RBBB is associated with an increased risk of NAFLD in young healthy individuals. Int J Cardiol 2013;168:4056-4057. doi: 10.1016/j.ijcard.2013. 07.035.

[29] Pokorney SD, Radder C, Schulte PJ, Al-Khatib SM, Tricocci P, Van de Werf F, et al. High-degree atrioventricular block, asystole, and electro-mechanical dissociation complicating non-ST-segment elevation myocardial infarction. Am Heart J 2016;171:25-32. doi: 10.1016/j.ahj.2015.09.004.

[30] Auffret V, Loirat A, Leurent G, Martins RP, Filippi E, Coudert I, et al. Highdegree atrioventricular block complicating ST segment elevation myocardial infarction in the contemporary era. Heart 2016;102:40-49. doi: 10.1136/ heartjnl-2015-308260.

[31] Coumbe AG, Naksuk N, Newell MC, Somasundaram PE, Benditt DG, Adabag S. Long-term follow-up of older patients with Mobitz type I second degree atrioventricular block. Heart 2013;99:334-338. doi: 10.1136/heartjnl2012-302770.

[32] Crisel RK, Farzaneh-Far R, Na B, Whooley MA. First-degree atrioventricular block is associated with heart failure and death in persons with stable coronary artery disease: data from the Heart and Soul Study. Eur Heart J 2011; 32:1875-1880. doi: 10.1093/eurheartj/ehr139.

[33] Kwok CS, Rashid M, Beynon R, Barker D, Patwala A, Morley-Davies A, et al. Prolonged PR interval, first-degree heart block and adverse cardiovascular outcomes: a systematic review and meta-analysis. Heart 2016;102:672680. doi: 10.1136/heartjnl-2015-308956.

[34] Imanishi R, Seto S, Ichimaru S, Nakashima E, Yano K, Akahoshi M. Prognostic significance of incident complete left bundle branch block observed over a 40-year period. Am J Cardiol 2006;98:644-648. doi: 10.1016/j.amjcard. 2006.03.044.

[35] Bussink BE, Holst AG, Jespersen L, Deckers JW, Jensen GB, Prescott E. Right bundle branch block: prevalence, risk factors, and outcome in the general population: results from the Copenhagen City Heart Study. Eur Heart J 2013; 34:138-146. doi: 10.1093/eurheartj/ehs291.

[36] Zhang ZM, Rautaharju PM, Prineas RJ, Loehr L, Rosamond W, Soliman EZ. Ventricular conduction defects and the risk of incident heart failure in the Atherosclerosis Risk in Communities (ARIC) Study. J Card Fail 2015;21: 307-312. doi: 10.1016/j.cardfail.2015.01.001.

[37] Anstee QM, Targher G, Day CP. Progression of NAFLD to diabetes mellitus, cardiovascular disease or cirrhosis. Nat Rev Gastroenterol Hepatol 2013;10: 330-344. doi: 10.1038/nrgastro.2013.41.

[38] Byrne CD, Targher G. NAFLD: a multisystem disease. J Hepatol 2015;62: S47-S64. doi: 10.1016/j.jhep.2014.12.012.

[39] Lonardo A, Ballestri S, Targher G, Loria P. Diagnosis and management of cardiovascular risk in nonalcoholic fatty liver disease. Expert Rev Gastroenterol Hepatol 2015;9:629-650. doi: 10.1586/17474124.2015.965143.

[40] Byrne CD, Targher G. Ectopic fat, insulin resistance, and nonalcoholic fatty liver disease: implications for cardiovascular disease. Arterioscler Thromb Vasc Biol 2014;34:1155-1161. doi: 10.1161/ATVBAHA.114.303034.

[41] Targher G, Byrne CD. Diagnosis and management of nonalcoholic fatty liver disease and its hemostatic/thrombotic and vascular complications. Semin Thromb Hemost 2013;39:214-228. doi: 10.1055/s-0033-1334866.

[42] Loria P, Carulli L, Bertolotti M, Lonardo A. Endocrine and liver interaction: the role of endocrine pathways in NASH. Nat Rev Gastroenterol Hepatol 2009;6: 236-247. doi: 10.1038/nrgastro.2009.33.

[43] Hallsworth K, Hollingsworth KG, Thoma C, Jakovljevic D, MacGowan GA, Anstee QM, et al. Cardiac structure and function are altered in adults with 
non-alcoholic fatty liver disease. J Hepatol 2013;58:757-762. doi: 10 . 1016/j.jhep.2012.11.015.

[44] VanWagner LB, Wilcox JE, Colangelo LA, Lloyd-Jones DM, Carr JJ, Lima JA, et al. Association of nonalcoholic fatty liver disease with subclinical myocardial remodeling and dysfunction: A population-based study. Hepatology 2015;62:773-783. doi: 10.1002/hep.27869.

[45] Mantovani A, Pernigo M, Bergamini C, Bonapace S, Lipari P, Pichiri I, et al. Nonalcoholic fatty liver disease is independently associated with early left ventricular diastolic dysfunction in patients with type 2 diabetes. PLoS One 2015;10:e0135329. doi: 10.1371/journal.pone.0135329.

[46] Markus MR, Baumeister SE, Stritzke J, Dörr M, Wallaschofski H, Völzke H, et al. Hepatic steatosis is associated with aortic valve sclerosis in the general population: the Study of Health in Pomerania (SHIP). Arterioscler Thromb Vasc Biol 2013;33:1690-1695. doi: 10.1161/ATVBAHA.112.300556.

[47] Bonapace S, Valbusa F, Bertolini L, Pichiri I, Mantovani A, Rossi A, et al. Nonalcoholic fatty liver disease is associated with aortic valve sclerosis in patients with type 2 diabetes mellitus. PLoS One 2014;9:e88371. doi: 10. 1371/journal.pone.0088371.

[48] Mantovani A, Pernigo M, Bergamini C, Bonapace S, Lipari P, Valbusa F, et al. Heart valve calcification in patients with type 2 diabetes and nonalcoholic fatty liver disease. Metabolism 2015;64:879-887. doi: 10.1016/j.metabol. 2015.04.003.

[49] Newton JL, Pairman J, Wilton K, Jones DE, Day C. Fatigue and autonomic dysfunction in non-alcoholic fatty liver disease. Clin Auton Res 2009;19: 319-326. doi: 10.1007/s10286-009-0031-4. 